\title{
Generalized Onsager theory of liquid crystals
}

\author{
Xiaobin Xiao and Ping Sheng \\ Department of Physics, Hong Kong University of Science and Technology, Clear Water Bay, Kowloon, Hong Kong, China
}

(Received 19 August 2013; published 2 December 2013)

\begin{abstract}
The Onsager theory is known to be inaccurate in its prediction of the critical transition density for small aspect ratio hard rods. In this paper we generalize the Onsager theory in two dimensions by taking into account the short-range order as well as the higher-order virial coefficients, up to the fourth order. By carrying out molecular dynamics (MD) simulations on "molecules" comprising linked hard disks with an aspect ratio $\ell$ ranging from 5 to 13 , we show that the generalized theory is much improved as compared to the traditional theory, with its predictions of the transition density agreeing well with the simulation results. This indicates the importance of short-range order considerations (in conjunction with steric repulsion) for molecules with $\ell \leqslant 10$, a group which includes the most commonly encountered thermotropic liquid crystals. MD simulations further yield evidence for hexagonal order for molecules with $\ell \leqslant 8$, indicating an intermediate hexagonal phase before solidifying at higher densities.
\end{abstract}

DOI: 10.1103/PhysRevE.88.062501

PACS number(s): 64.70.M-, 61.30.Cz, 61.20.Ja

\section{INTRODUCTION}

First among all the liquid crystal (LC) theories is the theory of Onsager [1-6]. This is not only because it is the first theory to show that orientational order can arise from molecular shape anisotropy, but also because of its subsequent influence in diverse areas of physics and applied mathematics. Onsager treated liquid crystal molecules as interacting hard rods in which the free energy can be expanded as virial coefficients, in terms of the orientational distribution function. For a system of $N$ rods in a volume $V$ at temperature $T$, the free energy can be calculated to the first order of density as

$$
\begin{aligned}
\frac{F}{N k_{B} T}= & -\frac{\ln Z}{N}=\ln \left(\rho \eta \lambda^{3}\right)+\int d \Omega f(\Omega) \ln f(\Omega) \\
& -\frac{\rho}{2} \iint d \Omega_{1} d \Omega_{2} \beta_{1}\left(\Omega_{1}, \Omega_{2}\right) f\left(\Omega_{1}\right) f\left(\Omega_{2}\right) .
\end{aligned}
$$

Here $F$ denotes the free energy, $k_{B}$ the Boltzmann constant, $Z$ the partition function, $\rho$ the number density, $f$ the angular (orientational) distribution function, $\Omega$ the solid angle, and $\lambda$ and $\eta$ are constants which do not play a role in the theory. By considering only a steric repulsive interaction between the rods, the second virial coefficient $\beta_{1}$ is simply the excluded volume of two rods. By using trial orientational distribution functions, the free energy can be minimized to obtain the orientational state of the rods at any given density. In three dimensions, the Onsager theory qualitatively predicts a first-order isotropic-nematic transition (second order in two dimensions), but the transition density is too low and the density discontinuity is too large compared with the experiments and simulations. Moreover, since only the steric interaction is considered, the transition is entirely independent of the temperature.

The shortcoming of the Onsager theory does not lie in its basic approach, which is rigorous, but rather in its omissions and approximation. The first approximation is the consideration of only a steric repulsive interaction. Under this approximation, the theory is only sensitive to density variations, with no temperature dependence. The second approximation can be stated as follows: "There is no short-range order apart from the long-range order.' In other words, if we describe the orientational distribution of two nearest-neighbor molecules, labeled $A$ and $B$, by $f^{(2)}\left(\theta_{a}, \theta_{b}\right)$, where the superscript " (2)" means that the distribution function is a two-particle distribution function and $\theta_{a(b)}$ denotes the polar orientation angle of molecule $A(B)$ relative to a laboratory coordinate; then Onsager has made the approximation by writing $f^{(2)}\left(\theta_{a}, \theta_{b}\right) \cong f^{(1)}\left(\theta_{a}\right) f^{(1)}\left(\theta_{b}\right)$. In a homogeneous system, we do not expect $f^{(1)}\left(\theta_{a}\right)$ to differ from $f^{(1)}\left(\theta_{b}\right)$; hence they both denote the (same) long-range orientational order, i.e., short-range order does not play a role in the Onsager theory. Originally intended for rodlike molecules with very large aspect ratios, the theory predicts in three dimensions (3D) a strong first-order orientational phase transition [and a continuous transition in two dimensions (2D)] as the density is varied, with a large density change. Although there is no rigorous proof, the suspicion has always been that the Onsager theory is indeed valid in the large aspect ratio limit, but it is well known that its prediction is inaccurate in the small aspect ratio regime [7], e.g., aspect ratio less than 8-10, the group which can encompass some of the most commonly encountered thermotropic nematic liquid crystal molecules.

In this paper we generalize the Onsager theory along two directions. First, short-range order will be included through the consideration of the two-body distribution function. This is described in Sec. II. Second, by including the higherorder virial coefficients, we intend to make the theory more accurate beyond the low density limit. Owing to the very significant multidimensional calculations involved, we shall limit ourselves to two spatial dimensions (2D), with the hard-core steric repulsive interactions between the molecules. Here a "molecule" with aspect ratio $\ell$ is modeled by $\ell$ hard disks of diameter 1 , rigidly linked together with their centers along a straight line. This aspect is detailed in Sec. III. To obtain the thermodynamic properties of the system requires minimizing the total free energy. This is described in Sec. IV. The effect of the short-range order is checked by obtaining the theory predictions with and without the short-range order. It is seen that without the short-range order, including the higher-order virial coefficients actually makes the predictions even worse than the original Onsager theory, i.e., the predicted 
isotropic-nematic transition density becomes much lower than that seen in the simulations, and increasingly so with increasing orders of the virial coefficients. In Sec. V we compare the predictions of the generalized theory with the results of molecular dynamics (MD) simulations. It is shown that not only does the nematic-isotropic transition move to higher densities with the inclusion of the short-range order, but the accuracy of the theory prediction is also extended to smaller aspect ratio molecules. We conclude in Sec. VI by noting some of the implications and the potential for further works.

\section{SHORT-RANGE ORDER}

Short-range orientational order in liquid crystals is defined by the correlation in the orientations of the neighboring rodlike molecules. While known to exist and even observed either through experiments or simulations [7-14], short-range order has been ignored in most LC theories and is by itself a somewhat controversial subject with regard to how strong the effect can be, thermodynamically. However, its introduction into the problem can enrich the theory. In particular, it becomes theoretically possible to have an orientationally isotropic state with strong short-range order but without long-range orientational order. Such considerations have been absent in the most popular theories such as the Landau-de Gennes theory, the Onsager theory, and the Maier-Saupe theory.

Spatially extended short-range orientational order with an added degree of thermodynamic stability is sometimes also known as cybotactic clusters [7,11-16] (i.e., a group of molecules with the same alignment). The term cybotactic clusters has been used in particular to denote the pretransitional fluctuation effect above the smectic liquid crystalline phase, although it has also been used more loosely to denote more transient short-range orderings that may appear in the isotropic phase of nematic liquid crystals.

From our simulation results as shown in Fig. 1, short-range order in the form of cybotactic clusters can be important in those systems in which the aspect ratio of the anisotropic molecules is less than 6-8 (here the aspect ratio is 5). For large aspect ratios such clusters may be more difficult to identify, although short-range order may still exist. The state shown in Fig. 1 obviously has a lower free energy (compared to the state of overall long-range orientational order), owing to higher orientational entropy. In fact, in this particular case the Onsager theory's prediction is wrong, and this fact has been known previously [7]. However, all it means is that the Onsager theory probably should never be applied to molecules with such a small aspect ratio (5 in this case).

Short-range orientational order introduces a mesoscale into the problem - the short-range correlation length. In the case of cybotactic clusters, its scale is roughly the aspect ratio $\ell$. Its consideration can potentially reconcile the theory to the small density change at the nematic-isotropic transition because the density is a local characteristic and hence is strongly coupled to short-range orientational order and is only weakly coupled to long-range orientational order. The appearance of the latter is of course the criterion for the nematic order. This view was proposed by some earlier works $[8,9]$.

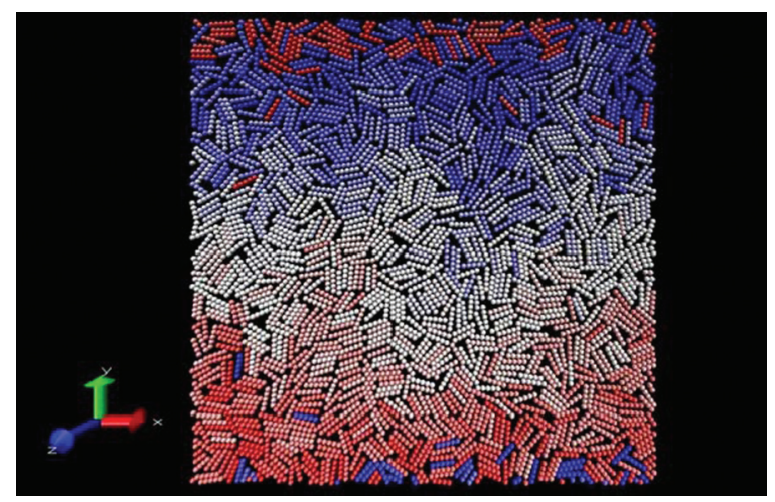

FIG. 1. (Color online) Simulation results for molecules with aspect ratio 5. Strong short-range order, in the form of 2D cybotatic clusters, is clearly seen. The cluster size is determined by the aspect ratio. Such strong short-range order is especially significant for the short aspect ratio molecules. Through strong short-range ordering, the clusters have a reduced aspect ratio as compared to individual molecules. Such an arrangement clearly has higher entropy than a state with long-range orientational order.

In this context, other theories which should be mentioned are as follows. By adopting the cluster variational approach, Sheng and Wojtowicz developed a constant coupling theory of nematics [17] that is an extension of the Maier-Saupe theory by including some short-range order. Tao, Sheng, and Lin also extended the mean field theory of the isotropic-nematic (I-N) transition by the inclusion of the isotropic, density-dependent component of the molecular interaction. The magnitude of $\left(T_{c}-T^{*}\right) / T_{c}$ and the density change at the transition agreed well with the experiments [18]. More recently, a mean field type of theory for nematics, which includes cybotactic shortrange ordering, has been proposed [12].

In $2 \mathrm{D}$, the Onsager theory may be written as

$$
\begin{aligned}
\frac{F}{N k_{B} T}= & -\frac{\ln Z}{N}=\ln \left(\rho \eta \lambda^{2}\right)+\int d \theta f(\theta) \ln f(\theta) \\
& -\frac{\rho}{2} \iint d \theta_{a} d \theta_{b} \beta_{1}\left(\theta_{a}, \theta_{b}\right) f\left(\theta_{a}\right) f\left(\theta_{b}\right),
\end{aligned}
$$

where $\theta$ denotes the angle with respect to the $z$ axis of the planar laboratory coordinate, and $f(\theta)$ without the superscript implicitly implies a one-body distribution function. In the Onsager theory the approximation $f^{(2)}\left(\theta_{a}, \theta_{b}\right) \cong$ $f^{(1)}\left(\theta_{a}\right) f^{(1)}\left(\theta_{b}\right)$ was used. However, it is well known that one can write $f^{(2)}\left(\theta_{a}, \theta_{b}\right)$ alternatively as $f^{(2)}\left(\theta_{a}, \theta_{b}\right)=$ $f^{(1)}\left(\theta_{a}\right) h\left(\theta_{b} \mid \theta_{a}\right)$, where $h\left(\theta_{b} \mid \theta_{a}\right)$ has the meaning of the orientational distribution of particle $B$, given that particle $A$ is oriented at $\theta_{a}$ relative to the laboratory coordinate. Here $h$ has exactly the meaning of short-range order, since it depends only on the relative orientation between particles $A$ and $B$.

To introduce short-range order into the entropy term, let us start with $\int d \theta_{a} f\left(\theta_{a}\right) \ln f\left(\theta_{a}\right)$, the orientational entropy term in the Onsager theory. It is clear that we can rewrite it 
as follows:

$$
\begin{aligned}
& \int d \theta_{a} f\left(\theta_{a}\right) \ln f\left(\theta_{a}\right) \\
& =\frac{1}{2} \int d \theta_{a} \int d \theta_{b} f\left(\theta_{a}\right) f\left(\theta_{b}\right) \ln \left[f\left(\theta_{a}\right) f\left(\theta_{b}\right)\right] \\
& \cong \frac{1}{2} \int d \theta_{a} \int d \theta_{b} f^{(2)}\left(\theta_{a}, \theta_{b}\right) \ln \left[f^{(2)}\left(\theta_{a}, \theta_{b}\right)\right] \\
& =\frac{1}{2} \int d \theta_{a} \int d \theta_{b} f\left(\theta_{a}\right) h\left(\theta_{b} \mid \theta_{a}\right) \ln \left[f\left(\theta_{a}\right) h\left(\theta_{b} \mid \theta_{a}\right)\right] \\
& =\frac{1}{2} \int d \theta_{a} f\left(\theta_{a}\right) \ln f\left(\theta_{a}\right) \\
& \quad+\frac{1}{2} \int d \theta_{b} h\left(\theta_{b} \mid \theta_{a}\right) \ln h\left(\theta_{b} \mid \theta_{a}\right) .
\end{aligned}
$$

The two molecules $A$ and $B$ should be nearest neighbors, since otherwise in the virial coefficient calculations they do not interact. We treat $h\left(\theta_{b} \mid \theta_{a}\right)$ to be independent of the distance between the molecules $A$ and $B$. It can be regarded as some proper average of the correlation functions within the correlation length. As $h\left(\theta_{b} \mid \theta_{a}\right)$ is dependent only on the relative angle $\Delta \theta$ between the neighboring $A$ and $B$, we can write $h\left(\theta_{b} \mid \theta_{a}\right)=h(\Delta \theta)$ below. It should be noted that that there are always the normalization conditions of the distribution functions:

$$
\begin{aligned}
\int_{0}^{2 \pi} f\left(\theta_{a}\right) d \theta_{a} & =1, \\
\int_{0}^{2 \pi} h(\Delta \theta) d(\Delta \theta) & =1 .
\end{aligned}
$$

Equation (2) can now be rewritten as

$$
\begin{aligned}
\frac{F}{N k_{B} T}= & \frac{1}{2} \int d \theta f(\theta) \ln f(\theta) \\
& +\frac{1}{2} \int d(\Delta \theta) h(\Delta \theta) \ln h(\Delta \theta) \\
& -\frac{\rho}{2} \int d(\Delta \theta) \beta_{1}(\Delta \theta) h(\Delta \theta) .
\end{aligned}
$$

In Eq. (4) we have taken note of the fact that $\beta_{1}$, the second virial coefficient, depends only on the relative angle between the two molecules, hence the integration of $f\left(\theta_{a}\right)$ can be immediately carried out (to yield 1).

In anticipation of adding higher-order virial coefficients to Eq. (4), one can expect that there can be terms such as $f^{(3)}\left(\theta_{a}, \theta_{b}, \theta_{c}\right) \beta_{2}\left(\theta_{a}, \theta_{b}, \theta_{c}\right)$ and $f^{(4)}\left(\theta_{a}, \theta_{b}, \theta_{c}, \theta_{d}\right) \beta_{3}\left(\theta_{a}, \theta_{b}, \theta_{c}, \theta_{d}\right)$, the three-body distribution function and four-body distribution function multiplied by their respective third and fourth virial coefficient. For the entropy term, one can write for $f^{(3)}\left(\theta_{a}, \theta_{b}, \theta_{c}\right)$ the following approximate expression:

$$
\begin{aligned}
\int d \theta_{a} f\left(\theta_{a}\right) \ln f\left(\theta_{a}\right) & =\frac{1}{3} \int d \theta_{a} \int d \theta_{b} \int d \theta_{c} f\left(\theta_{a}\right) f\left(\theta_{b}\right) f\left(\theta_{c}\right) \ln \left[f\left(\theta_{a}\right) f\left(\theta_{b}\right) f\left(\theta_{c}\right)\right] \\
& \cong \frac{1}{3} \int d \theta_{a} \int d \theta_{b} \int d \theta_{c} f^{(3)}\left(\theta_{a}, \theta_{b}, \theta_{c}\right) \ln \left[f^{(3)}\left(\theta_{a}, \theta_{b}, \theta_{c}\right)\right] \\
& =\frac{1}{3} \int d \theta_{a} \int d \theta_{b} \int d \theta_{c} f\left(\theta_{a}\right) h(\Delta \theta) f\left(\theta_{c}\right) \ln \left[f\left(\theta_{a}\right) h(\Delta \theta) f\left(\theta_{c}\right)\right] \\
& =\frac{2}{3} \int d \theta_{a} f\left(\theta_{a}\right) \ln f\left(\theta_{a}\right)+\frac{1}{3} \int d \Delta \theta h(\Delta \theta) \ln h(\Delta \theta),
\end{aligned}
$$

where $f^{(3)}\left(\theta_{a}, \theta_{b}, \theta_{c}\right)=f\left(\theta_{a}\right) h\left(\theta_{b}-\theta_{a}\right) f\left(\theta_{c}\right)$ instead of $f^{(2)}\left(\theta_{a}, \theta_{b}\right)$ in the third line of Eq. (5). It can be seen that such a replacement results in a change of coefficients $(2 / 3$ and $1 / 3$, instead of $1 / 2$ and $1 / 2)$ in front of the two terms in the last line of Eq. (5). For the four-particle distribution function, a similar treatment leads to the respective coefficients of 3/4 and 1/4. Since we have virial coefficients up to the fourth order, we shall treat the entropy term as

$$
\chi \int d \theta f(\theta) \ln f(\theta)+(1-\chi) \int d(\Delta \theta) g(\Delta \theta) \ln g(\Delta \theta),
$$

where $\chi$ is a parameter whose value falls between 0.5 and 0.75 . The two limiting values represent the upper and lower bounds on the importance of the short-range order contribution to entropy in our theory.

By adding short-range order, the free energy of the generalized Onsager theory has the following form:

$$
\begin{aligned}
F / N k T= & \ln \left(\rho \eta \lambda^{2}\right)-\ln (2 \pi)-1+\chi \int d \theta f(\theta) \ln f(\theta)+(1-\chi) \int d \Delta \theta h(\Delta \theta) \ln h(\Delta \theta) \\
& -\frac{1}{2} \rho \iint d \theta_{a} d \theta_{b} \beta_{1}\left(\theta_{a}, \theta_{b}\right) f\left(\theta_{a}\right) h\left(\theta_{a}-\theta_{b}\right) \\
& -\frac{1}{3} \rho^{2} \iiint d \theta_{a} d \theta_{b} d \theta_{c} \beta_{2}\left(\theta_{a}, \theta_{b}, \theta_{c}\right) f\left(\theta_{a}\right) f\left(\theta_{b}\right) h\left(\theta_{c}-\theta_{a}\right) \\
& -\frac{1}{4} \rho^{3} \iiint \int d \theta_{a} d \theta_{b} d \theta_{c} d \theta_{d} \beta_{3}\left(\theta_{a}, \theta_{b}, \theta_{c}, \theta_{d}\right) f\left(\theta_{a}\right) f\left(\theta_{b}\right) f\left(\theta_{c}\right) h\left(\theta_{d}-\theta_{a}\right)-\cdots
\end{aligned}
$$


Since the pressure $P=-\frac{\partial F}{\partial V}=\frac{\rho^{2}}{N} \frac{\partial F}{\partial \rho}$, the equation of state may be obtained from the free energy as

$$
\begin{aligned}
\frac{P}{k T}= & \rho\left[1-\frac{1}{2} \rho \iint d \theta_{a} d \theta_{b} \beta_{1}\left(\theta_{a}, \theta_{b}\right) f\left(\theta_{a}\right) h\left(\theta_{a}-\theta_{b}\right)-\frac{2}{3} \rho^{2} \iiint d \theta_{a} d \theta_{b} d \theta_{c} \beta_{2}\left(\theta_{a}, \theta_{b}, \theta_{c}\right) f\left(\theta_{a}\right) f\left(\theta_{b}\right) h\left(\theta_{c}-\theta_{a}\right)\right. \\
& \left.-\frac{3}{4} \rho^{3} \iiint \int d \theta_{a} d \theta_{b} d \theta_{c} d \theta_{d} \beta_{3}\left(\theta_{a}, \theta_{b}, \theta_{c}, \theta_{d}\right) f\left(\theta_{a}\right) f\left(\theta_{b}\right) f\left(\theta_{c}\right) h\left(\theta_{d}-\theta_{a}\right)-\cdots\right],
\end{aligned}
$$

where $\rho=N / V$ is the number density, $\eta$ is a dimensionless constant, and $\lambda$ is the thermal wavelength. $0.5<\chi<0.75$ is the parameter which adjusts the entropy contribution of the short-range order. It will be seen below that the simulation results fall between the theory predictions using the upper and lower bound values of $\chi$.

\section{VIRIAL COEFFICIENTS}

Virial coefficients are the irreducible parts of the cluster integrals, which in turn are the coefficients of the cluster expansion for the equation of state [19]. For 2D anisotropic molecules with fixed orientations, the respective virial coefficients up to the fourth order are given by

$$
\begin{gathered}
\beta_{1}\left(\theta_{1}, \theta_{2}\right)=\frac{1}{A} \iint_{A} d \vec{r}_{1} d \vec{r}_{2} \Phi_{12}\left(\vec{r}_{1}, \vec{r}_{2}, \theta_{1}, \theta_{2}\right) \\
\beta_{2}\left(\theta_{1}, \theta_{2}, \theta_{3}\right)=\frac{1}{2 A} \iiint d \vec{r}_{1} d \vec{r}_{2} d \vec{r}_{3} \Phi_{12} \Phi_{13} \Phi_{23} \\
\beta_{3}\left(\theta_{1}, \theta_{2}, \theta_{3}, \theta_{4}\right)=\frac{1}{6 A}\left[3 \times\left(v c_{3}^{1}\right)+6 \times\left(v c_{3}^{2}\right)+\left(v c_{3}^{3}\right)\right] \\
v c_{3}^{1}=\iiint_{\vec{r}_{1}, \vec{r}_{2}, \vec{r}_{3}, \vec{r}_{4}} d \vec{r}_{1} d \vec{r}_{2} d \vec{r}_{3} d \vec{r}_{4} \Phi_{14} \Phi_{24} \Phi_{31} \Phi_{32} \\
v c_{3}^{2}=\iiint_{\vec{r}_{1}, \vec{r}_{2}, \vec{r}_{3}, \vec{r}_{4}} d \vec{r}_{1} d \vec{r}_{2} d \vec{r}_{3} d \vec{r}_{4} \Phi_{14} \Phi_{24} \Phi_{34} \Phi_{12} \Phi_{23} \\
v c_{3}^{3}=\iiint_{\vec{r}_{1}, \vec{r}_{2}, \vec{r}_{3}, \vec{r}_{4}} d \vec{r}_{1} d \vec{r}_{2} d \vec{r}_{3} d \vec{r}_{4} \Phi_{14} \Phi_{24} \Phi_{34} \Phi_{12} \Phi_{23} \Phi_{31},
\end{gathered}
$$

where $\quad \Phi_{12}\left(x, y, \theta_{1}, \theta_{2}\right)=\exp \left[-v_{12}\left(x, y, \theta_{1}, \theta_{2}\right) / k_{B} T\right]-1$, $v_{12}\left(x, y, \theta_{1}, \theta_{2}\right)$ denotes the two-body interaction potential between two molecules, $d \vec{r}=d x d y, \vec{r}=(x, y)$, and we have used 1, 2, 3, 4 instead of $a, b, c, d$ to denote the molecules. For the hard-core steric repulsive potential, $\Phi_{12}=0$ when two molecules do not overlap, and $\Phi_{12}=-1$ in spatial regions where there is overlap. The Onsager theory for molecules interacting with the Gay-Berne potential has been studied by Ginzburg, Glaser, and Clark [20].

In this paper we consider a 2D model of anisotropic molecules, each comprising $\ell$ nonintersecting, touching hard disks with radius 1 , linked together with their centers aligned rigidly along a straight line. The disks are "hard" in the sense of having infinite positive interaction energy if they intersect. The molecules described above have an aspect ratio $\ell$.
In two-dimensional systems, the fourth virial coefficient is a ten-dimensional integral, impossible for the usual integration method. In this project we choose to use the Monte Carlo integration approach since this method is less sensitive to the dimensionality of the integral. The Monte Carlo integration method is faster than ordinary integration when the dimension of the integral is larger than 4. Since the trials of the Monte Carlo method are independent of each other, the parallel code for the Monte Carlo integration can be very efficient. We have carried out the numerical evaluation of the virial coefficients and stored them in computer memory for the purpose of freeenergy evaluations.

It should be mentioned that in 2D, the true picture of a phase transition is the change in the behavior of the molecular correlation function, from exponential decay as a function of separation below the transition to quasi-long-range order characterized by power-law decay above the transition [21]. However, in the present case, with a limited number of virial coefficients, and hence a limited range of molecular separations, it is impossible to delineate such a phase transition picture. It will be shown that in spite of such limitations, the incorporation of short-range order, in conjunction with the higher-order virial coefficients, can still reasonably predict the transition density even at the small aspect ratio regime.

\section{FREE-ENERGY MINIMIZATION}

In previous comparisons between the Onsager theory predictions and simulations, the Onsager theory generally predicts a lower phase transition density than that observed in simulations. Hence it is desirable to see whether the extension of the virial coefficients to higher order can improve the situation. However, that requires the minimization of the free energy, given by Eq. (7). For this purpose, we expand the one-body distribution function as

$$
\begin{gathered}
f(\theta)=\frac{1}{2 \pi}\left[1+\sum_{n=1}^{\infty} a_{2 n} \cos (2 n \theta)\right], \\
h(\Delta \theta)=\frac{1}{2 \pi}\left[1+\sum_{n=1}^{\infty} b_{2 n} \cos (2 n \Delta \theta)\right] .
\end{gathered}
$$

By substituting Eqs. (9) and (10) into Eq. (7), the free energy becomes a function of the set of scalar parameters $\left\{a_{2 n}, b_{2 n}\right\}$. It is noted that only the even order appears, owing to the symmetry of the molecules, i.e., there is no difference between the heads and tails. Also, from Eqs. (9) and (10) it is easy to see that $a_{2 n}, b_{2 n} \leqslant 2$. 

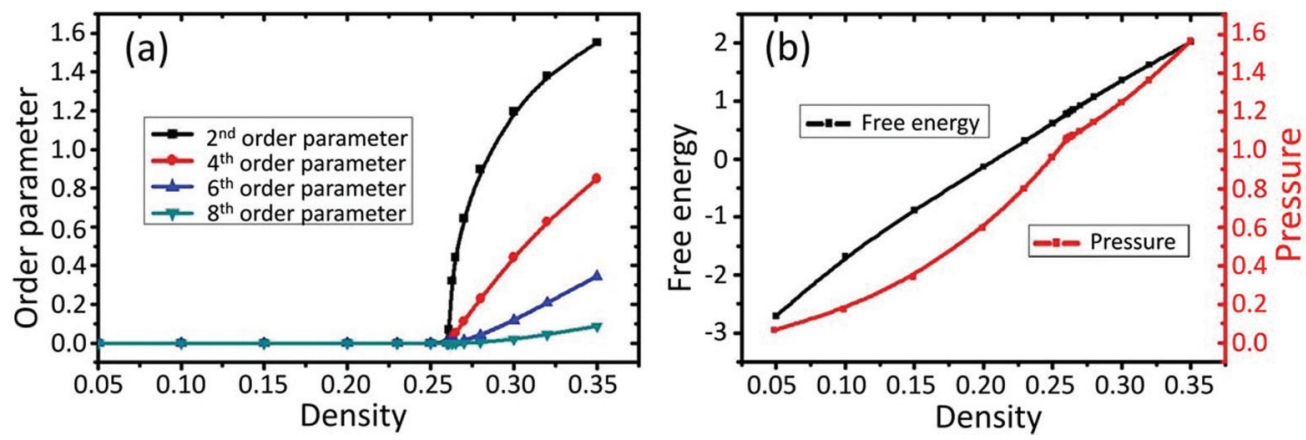

FIG. 2. (Color online) Isotropic-nematic transition for molecules with aspect ratio $\ell=9$, predicted by the Onsager theory with the inclusion of virial coefficents up to the fourth order, but without the consideration of short-range order. (a) The order parameters $a_{2}, a_{4}, a_{6}$, and $a_{8}$ plotted as a function of density. (b) Free energy and pressure plotted as a function of density. The density is measured in units of $\rho_{o}$. There is a tiny kink in the free-energy curve, at around $\rho / \rho_{o}=0.26$. The kink is more visible in the pressure curve. In $2 \mathrm{D}$, the transition is second order. In this case the simulation results show the transition density isat $\rho / \rho_{o}=0.44$.

\section{A. Integration with the basis function}

We first perform the integration of the virial coefficients with the basis functions. Thus we have

$$
\begin{aligned}
& \int_{0}^{2 \pi} d \theta_{1} \int_{0}^{2 \pi} d \theta_{2} \beta_{1}\left(\theta_{1}, \theta_{2}\right) f\left(\theta_{1}\right) h\left(\theta_{2}-\theta_{1}\right) \\
& =B^{0}+\sum_{n=1}^{\infty} B_{2 n}^{1} b_{2 n},
\end{aligned}
$$

where

$$
\begin{aligned}
B^{0} & =\frac{1}{2 \pi} \int_{0}^{2 \pi} d \theta \beta_{1}(\theta), \\
B_{2 n}^{1} & =\frac{1}{2 \pi} \int_{0}^{2 \pi} d \theta \beta_{1}(\theta) \cos [2 n(\theta)] .
\end{aligned}
$$

It should be noted that up to the second virial coefficient, the $a_{2 n}$ 's that represent long-range order do not appear. That is clear from the fact that $\beta_{1}$ depends only on the relative orientation between two molecules, and with the introduction of short-range order only the $h(\Delta \theta)$ matters. $f\left(\theta_{1}\right)$ integrates to 1 . Hence in the generalized theory it is necessary to go to higher-order virial coefficients before the long-range order can be evaluated. Similar to the above, we can reduce the third- and fourth-order virial coefficients into expressions involving the summation of scalar coefficients and the results of integrals of the $\beta$ functions with the trigonometric functions. Details are given in Appendix A, where the expressions for the free energy and pressure, in terms of the coefficients, are also presented.

The state of the minimum free energy, together with its pressure versus density behavior, can be obtained by minimizing with respect to $\left\{a_{2 n}, b_{2 n}\right\}$. Since we are mainly interested in the behavior of $\left(a_{2}, a_{4}\right)$ and $\left(b_{2}, b_{4}\right)$, we retain terms up to $n=4$ in the numerical minimization.

\section{B. Results with and without short-range order}

We would like to check the importance of short-range order in the generalized theory. For this purpose we set $\chi=1$ in Eqs. (A13) and (A14), i.e., no short-range order, as well as express $f^{(n)}\left(\theta_{1}, \theta_{2},-\theta_{n}\right)=\Pi_{i=1,-n} f\left(\theta_{i}\right)$ in the virial coefficients. In this manner, we find that the inclusion of the higher-order virial coefficients actually lowers the transition density. This is seen in Figs. 2(a) and 2(b) for the case of molecules with aspect ratio $\ell=9$. In Fig. 2(a), it is clear that the isotropic-nematic transition occurs at a nondimensionalized density of $\rho / \rho_{o}=0.26$ when one considers virial coefficients up to the fourth order, where $\rho_{o}$ is the inverse of the volume (area) of a single molecule, $\rho_{o}=(\pi \ell / 4)^{-1}$. The simulation results (see below) have indicated a transition density of $\rho / \rho_{o}=0.44$. So the inclusion of the higher virial coefficients, without short-range order, actually does not help. In order to have a clear view of the trend, in Fig. 3 we show the transition densities plotted as a function of the aspect ratio, for the Onsager theory with inclusion of the second virial coefficient (the original theory), and the third and fourth virial coefficients. It is seen that the trend is a continuous downward shift, past the simulation value of 0.44 .

\section{Spatial dependence of short-range order}

Short-range order is usually a function of both relative orientation and spatial separation. However, in the present case the short-range order function $h$ is a function of the relative orientation only, between the neighboring molecules. This is

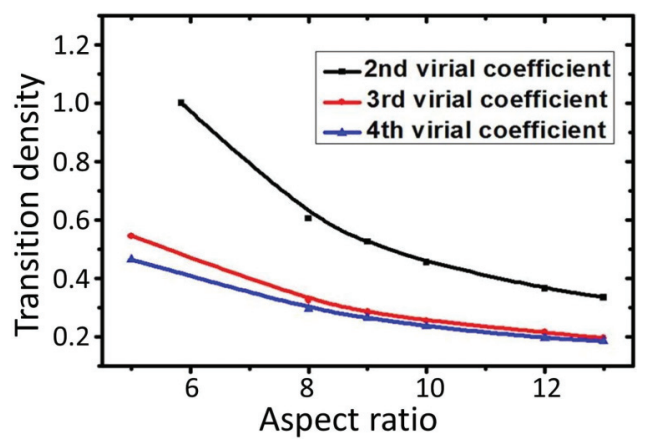

FIG. 3. (Color online) Transition density, measured in units of $\rho_{o}$, plotted as a function of the molecular aspect ratio, for the Onsager theory with the inclusion of virial coefficents up to the second, third, and fourth order, plotted as a function of the molecular aspect ratio. The simulation result, $\rho / \rho_{o}=0.44$ for aspect ratio 9 , lies between the black (square) and red (circle) curve. For the Onsager theory with only the second-order virial coefficient (black square curve), there is no nematic-isotropic transition for an aspect ratio of lower than 6 . 
because, within the context of the Onsager theory, the relative spatial separation is an integration variable in the calculation of the virial coefficient, hence it cannot appear explicitly. However, we can estimate the spatial dependence, over short separations and small $\Delta \theta$, by using the formula $f(\Delta \theta, n w)=$ $[h(\Delta \theta) / h(0)]^{n}, n \geqslant 1$. Here $f$ denotes the approximate shortrange order, and $w$ denotes the average separation between the centers of two molecules, which is constrained by the lower bound $w \geqslant 1+L \sin \Delta \theta$. The rationale underlying this formula is that the probability of having a relative orientational angle $\Delta \theta$ between two molecules separated by $n-1$ molecules in between is the product of the nearest-neighbor orientational short-range order. But this obviously can only be a rough estimate. A better formulation and approach to obtain the spatial dependence of the short-range order is presently under pursuit.

\section{MOLECULAR DYNAMICS (MD) SIMULATIONS}

It is always important to check the results of theory by either experiments or simulations. In the present case we wish to obtain the characteristics of the isotropic-nematic phase transition from the MD simulations, to be compared with the predictions of the generalized Onsager theory. The standard MD simulations are based on using Newton's law, $\vec{F}=m \vec{a}$, in discretized form, both spatially and temporally. There are now standard books [22,23] on this subject which one can follow to resolve the complications (such as maintaining a fixed temperature) of the discretization, therefore we will not provide details of the procedures here. However, for the steric repulsion part the MD simulation is not quite standard, although there are literature papers on the subject [24-26].

The complication of MD simulations by using a hard-core repulsive interaction is that the collision time is essentially zero. Hence one cannot use the discretized version of Newton's law to follow the trajectory. Instead, we have to use energy and (translational and angular) momenta conservation to determine the postcollision status. Details on the equations governing the molecular dynamics simulations are given in Appendix B.

\section{A. Orientational correlation function}

Phase transitions in 2D have been studied extensively, and it is known that (1) there can be no true long-range order-only quasi-long-range order exists in 2D, which means that the ordering decays in a power-law fashion as a function of separation distance, and (2) a 2D phase transition is characterized by a change in behavior from exponential decay of the correlation function to a power-law decay. In our MD simulations we therefore measure the following function:

$$
\alpha_{2 n}(r)=\langle\cos [2 n\{\theta(0)-\theta(r)\}]\rangle,
$$

where $\langle\cdots\rangle$ is a normalized average (as a function of time) over all pairs separated by a distance $r$, with one at the center and another falling within a circular annulus with chosen width $\Delta r$. For molecules with aspect ratio 9, the results are shown in Fig. 4 for the case of $n=1$.

Another interesting point one can see in Fig. 4 is that below the transition density, the decay of the orientational order becomes much steeper (i.e., exponential) when the separation

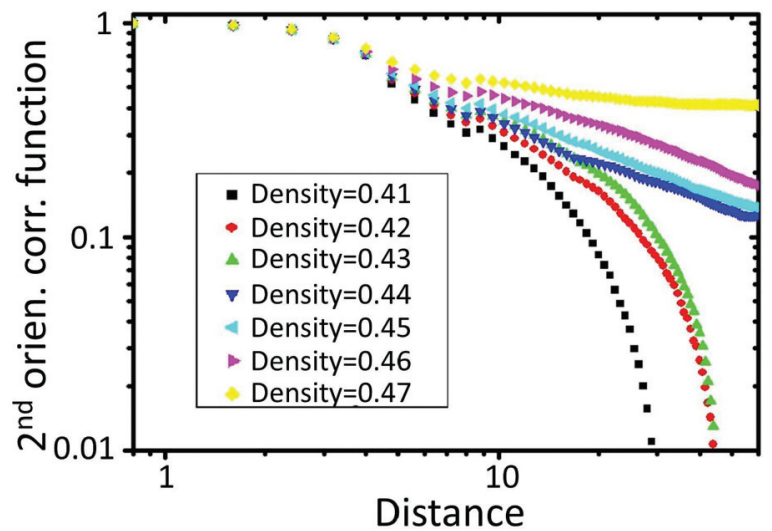

FIG. 4. (Color online) Orientational function plotted as a function of distance of separation, measured in units of the disk diameter, for the case of aspect ratio $\ell=9$. Since the plot is in a log-log scale, it is seen clearly that at a density of 0.44 the orientational correlation function acquires a power-law character. Hence $\rho / \rho_{o}=$ 0.44 is denoted the transition density. Distance is in units of hard disk diameter.

is larger than $\sim 9$. This is precisely the indication for the existence of cybostatic clusters whose overall aspect ratio is roughly on the order of the aspect ratio of the molecules.

\section{B. Comparison with the predictions of the generalized Onsager theory}

From simulations it is clear that since the orientational order is not a fixed constant, it would not be a good quantity to compare with the Onsager theory. However, the transition density is a rather robust quantity and hence can be meaningfully compared. In Fig. 5 we show that by using the upper and lower bounds of $\chi, 0.75$ and 0.5 , the predicted transition densities form a narrow band in which the simulation results are found. In Fig. 5 the red circle curve indicates the Onsager theory with the inclusion of up to the fourth-order virial coefficients. It lies much below the simulation results. Since the difference in the generalized theory and its original

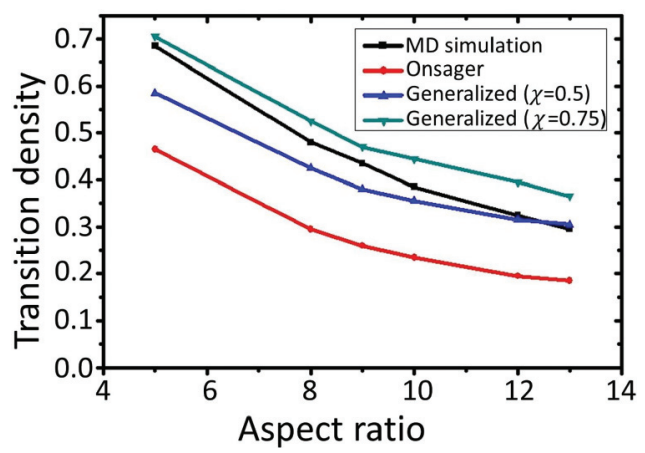

FIG. 5. (Color online) Comparison between the predicted transition density values, in units of $\rho_{o}$, and those observed in simulations, plotted as a function of the aspect ratio. It is seen that the simulation results fall right between the upper and lower bounds of the predicted values. The Onsager theory predictions, with the inclusion of up to the fourth-order virial coefficients, lie much lower than the simulation results. Hence the inclusion of the short-range order is the crucial element that enables the good agreement. 

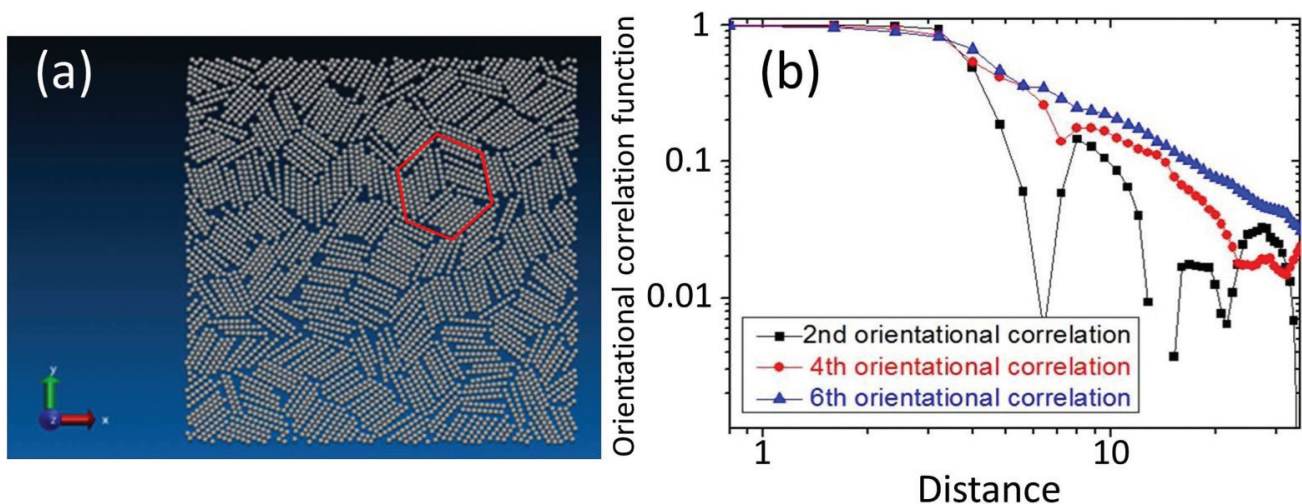

FIG. 6. (Color online) Hexatic order observed for the aspect ratio $\ell=8$ case, at a density of $\rho / \rho_{o}=0.64$. (a) A real space picture of the configuration. The red (circle) lines delineate a locally hexatic ordering. (b) The $n=3$ order parameter, the hexatic order (blue triangle symbols), is dominant over the lower-order parameters. Here the distance is measured in units of hard disk diameter. The hexatic order appears only within a very narrow density range. At $\rho / \rho_{o}=0.65$ it is already a solid, as characterized by a rather flat correlation function as a function of distance (measured in units of the hard disk diameter). At densities below $\rho / \rho_{o}=0.63$ the dominant order is $n=1$. The nematic-isotropic transition occurs at $\rho / \rho_{o}=0.50$. Sharp drops are observed at separations roughly corresponding to the aspect ratio times the diameter. The drops are due to the fact that at such separations one is very likely to be observing two molecules in different cybostatic clusters.

version lies in the consideration of short-range order, one can conclude that for those liquid crystal molecules with aspect ratios of less than 10 , the consideration of short-range order must constitute a very important element.

From Fig. 5 one can also extrapolate the simulation results to larger aspect ratio molecules. From the trend seen, it is speculated that the original Onsager theory can be accurate in predicting the transition density when the aspect ratio $\ell>20$. That is, the consideration of short-range order may not be physically relevant when the aspect ratio is large. One possible reason for this scenario is that the formation of cybostatic clusters is not favored since they are too costly in terms of the entropy. One can visualize from Fig. 1 that in order for there to be an easily identifiable cybostatic cluster, the ends of the molecules within a given cluster should align relatively well. However, for large aspect ratio molecules such an alignment would be very costly in terms of entropy.

\section{Observation of hexatic order}

Hexatic order is an inevitable consequence of the tight packing geometry in a system of hard spheres or disks [27-29]. When the molecules or particles deviate from the spherical geometry, it is expected that the hexatic order would not suddenly disappear. Instead, such an order should decrease in importance as the aspect ratio of the molecules increases. This is indeed the case observed in our MD simulation results as shown in Fig. 6(a). In particular, for $\ell=8$ the magnitude of the hexatic order $(n=3)$ is larger than the nematic order $(n=1)$. It is the dominant order as seen in Fig. 6(b). For aspect ratio $\ell=5$ the hexatic order is dominant over a much wider density range. In fact, the transition from the isotropic phase is directly into the hexatic phase, at $\rho / \rho_{o}=0.68$.

\section{CONCLUDING REMARKS}

The main point of this paper is to emphasize the importance of short-range order in small aspect ratio liquid crystalline molecular systems, together with an approach to introduce the short-range order into free-energy calculations. From the magnitude of the numerical effort required in the evaluation of the higher-order virial coefficients, the extension of the Onsager approach to 3D systems may not be feasible, especially if the attractive interaction is considered in conjunction with a steric repulsive interaction. However, our experience does suggest that while the steric interaction is the most important at the microscale, an attractive van der Waals interaction may operate on the meso- to macroscale. How to couple the interactions between these two scales should be the focus of future theories on liquid crystals.

\section{ACKNOWLEDGMENTS}

P.S. wishes to thank Tiezheng Qian and Chun Liu for useful discussions. This work is supported by RGC Grants No. HKUST/603512 and No. RPC11SC02.

\section{APPENDIX A: VIRIAL COEFFICIENTS, FREE ENERGY, AND PRESSURE EXPRESSIONS}

The third viral coefficient can be expressed as

$$
\begin{aligned}
& \int_{0}^{2 \pi} d \theta_{1} \int_{0}^{2 \pi} d \theta_{2} \int_{0}^{2 \pi} d \theta_{3} \beta_{2}\left(\theta_{1}, \theta_{2}, \theta_{3}\right) f\left(\theta_{1}\right) f\left(\theta_{2}\right) h\left(\theta_{3}-\theta_{1}\right) \\
& =C^{0}+\sum_{n=1}^{\infty} C_{2 n}^{1} b_{2 n}+\sum_{m=1}^{\infty} C_{2 m}^{2} a_{2 m} a_{2 m}+\sum_{m, n=1}^{\infty} C_{2 m, 2 n}^{3} a_{2 m} a_{m} b_{2 n}
\end{aligned}
$$


where

$$
\begin{gathered}
C^{0}=\left(\frac{1}{2 \pi}\right)^{3} \int_{0}^{2 \pi} d \theta_{1} \int_{0}^{2 \pi} d \theta_{2} \int_{0}^{2 \pi} d \theta_{3} \beta_{2}\left(\theta_{1}, \theta_{2}, \theta_{3}\right), \\
C_{2 n}^{1}=\left(\frac{1}{2 \pi}\right)^{3} \int_{0}^{2 \pi} d \theta_{1} \int_{0}^{2 \pi} d \theta_{2} \int_{0}^{2 \pi} d \theta_{3} \beta_{2}\left(\theta_{1}, \theta_{2}, \theta_{3}\right) \cos \left[2 n\left(\theta_{3}-\theta_{1}\right)\right], \\
C_{2 m}^{2}=\left(\frac{1}{2 \pi}\right)^{3} \int_{0}^{2 \pi} d \theta_{1} \int_{0}^{2 \pi} d \theta_{2} \int_{0}^{2 \pi} d \theta_{3} \beta_{2}\left(\theta_{1}, \theta_{2}, \theta_{3}\right) \cos \left(2 m \theta_{1}\right) \cos \left(2 m \theta_{2}\right), \\
C_{2 m, 2 n}^{3}=\left(\frac{1}{2 \pi}\right)^{3} \int_{0}^{2 \pi} d \theta_{1} \int_{0}^{2 \pi} d \theta_{2} \int_{0}^{2 \pi} d \theta_{3} \beta_{2}\left(\theta_{1}, \theta_{2}, \theta_{3}\right) \cos \left(2 m \theta_{1}\right) \cos \left(2 m \theta_{2}\right) \cos \left[2 n\left(\theta_{3}-\theta_{1}\right)\right] .
\end{gathered}
$$

For the fourth-order virial coefficient, we have

$$
\begin{aligned}
\int_{0}^{2 \pi} & d \theta_{1} \int_{0}^{2 \pi} d \theta_{2} \int_{0}^{2 \pi} d \theta_{3} \int_{0}^{2 \pi} d \theta_{4} \beta_{3}\left(\theta_{1}, \theta_{2}, \theta_{3}, \theta_{4}\right) f\left(\theta_{1}\right) f\left(\theta_{2}\right) f\left(\theta_{3}\right) h\left(\theta_{4}-\theta_{1}\right) \\
= & D^{0}+\sum_{n=1}^{\infty} D_{2 n}^{1} b_{2 n}+\sum_{n=1}^{\infty} D_{2 n}^{2} a_{2 n} a_{2 n}+\sum_{m, n, p=1}^{\infty} D_{2 m, 2 n, 2 p}^{3} a_{2 m} a_{2 n} a_{2 p}+\sum_{m, n=1}^{\infty} D_{2 m, 2 n}^{4} a_{2 m} a_{2 m} b_{2 n} \\
& +\sum_{m, n, p=1}^{\infty} D_{2 m, 2 n, 2 p, 2 q}^{5} a_{2 m} a_{2 n} a_{2 p} b_{2 q},
\end{aligned}
$$

where

$$
\begin{gathered}
D^{0}=\left(\frac{1}{2 \pi}\right)^{4} \int_{0}^{2 \pi} d \theta_{1} \int_{0}^{2 \pi} d \theta_{2} \int_{0}^{2 \pi} d \theta_{3} \int_{0}^{2 \pi} d \theta_{4} \beta_{3}\left(\theta_{1}, \theta_{2}, \theta_{3}, \theta_{4}\right) \\
D_{2 n}^{1}=\left(\frac{1}{2 \pi}\right)^{4} \int_{0}^{2 \pi} d \theta_{1} \int_{0}^{2 \pi} d \theta_{2} \int_{0}^{2 \pi} d \theta_{3} \int_{0}^{2 \pi} d \theta_{4} \beta_{3}\left(\theta_{1}, \theta_{2}, \theta_{3}, \theta_{4}\right) \cos \left[2 n\left(\theta_{4}-\theta_{1}\right)\right] \\
D_{2 n}^{2}=3\left(\frac{1}{2 \pi}\right)^{4} \int_{0}^{2 \pi} d \theta_{1} \int_{0}^{2 \pi} d \theta_{2} \int_{0}^{2 \pi} d \theta_{3} \int_{0}^{2 \pi} d \theta_{4} \beta_{3}\left(\theta_{1}, \theta_{2}, \theta_{3}, \theta_{4}\right) \cos \left(2 n \theta_{1}\right) \cos \left(2 n \theta_{2}\right) \\
D_{2 m, 2 n, 2 p}^{3}=\left(\frac{1}{2 \pi}\right)^{4} \int_{0}^{2 \pi} d \theta_{1} \int_{0}^{2 \pi} d \theta_{2} \int_{0}^{2 \pi} d \theta_{3} \int_{0}^{2 \pi} d \theta_{4} \beta_{3}\left(\theta_{1}, \theta_{2}, \theta_{3}, \theta_{4}\right) \cos \left(2 m \theta_{1}\right) \cos \left(2 n \theta_{2}\right) \cos \left(2 p \theta_{3}\right) \\
D_{2 m, 2 n}^{4}=2\left(\frac{1}{2 \pi}\right)^{4} \int_{0}^{2 \pi} d \theta_{1} \int_{0}^{2 \pi} d \theta_{2} \int_{0}^{2 \pi} d \theta_{3} \int_{0}^{2 \pi} d \theta_{4} \beta_{3}\left(\theta_{1}, \theta_{2}, \theta_{3}, \theta_{4}\right) \cos \left(2 m \theta_{1}\right) \cos \left(2 m \theta_{2}\right) \cos \left[2 n\left(\theta_{4}-\theta_{1}\right)\right] \\
+\left(\frac{1}{2 \pi}\right)^{4} \int_{0}^{2 \pi} d \theta_{1} \int_{0}^{2 \pi} d \theta_{2} \int_{0}^{2 \pi} d \theta_{3} \int_{0}^{2 \pi} d \theta_{4} \beta_{3}\left(\theta_{1}, \theta_{2}, \theta_{3}, \theta_{4}\right) \cos \left(2 m \theta_{2}\right) \cos \left(2 m \theta_{3}\right) \cos \left[2 n\left(\theta_{4}-\theta_{1}\right)\right] \\
D_{2 m, 2 n, 2 p, 2 q}^{5}=\left(\frac{1}{2 \pi}\right)^{4} \int_{0}^{2 \pi} d \theta_{1} \int_{0}^{2 \pi} d \theta_{2} \int_{0}^{2 \pi} d \theta_{3} \int_{0}^{2 \pi} d \theta_{4} \beta_{3}\left(\theta_{1}, \theta_{2}, \theta_{3}, \theta_{4}\right) \cos \left(2 m \theta_{1}\right) \cos \left(2 n \theta_{2}\right) \cos \left(2 p \theta_{3}\right) \cos \left[2 q\left(\theta_{4}-\theta_{1}\right)\right] .
\end{gathered}
$$

The resulting free energy is therefore given by

$$
\begin{aligned}
F / N k_{B} T= & \ln \rho+\frac{\chi}{2 \pi} \int_{0}^{2 \pi} d \theta\left[1+\sum_{n=1}^{\infty} a_{2 n} \cos (2 n \theta)\right] \ln \left[1+\sum_{n=1}^{\infty} a_{2 n} \cos (2 n \theta)\right] \\
& +\frac{1-\chi}{2 \pi} \int_{0}^{2 \pi} d \theta\left[1+\sum_{n=1}^{\infty} b_{2 n} \cos (2 n \theta)\right] \ln \left[1+\sum_{n=1}^{\infty} b_{2 n} \cos (2 n \theta)\right] \\
& -\frac{\rho}{2}\left[B^{0}+\sum_{n=1}^{\infty} B_{2 n}^{1} b_{2 n}\right]-\frac{\rho^{2}}{3}\left[C^{0}+\sum_{n=1}^{\infty} C_{2 n}^{1} b_{2 n}+\sum_{m=1}^{\infty} C_{2 m}^{2} a_{2 m} a_{2 m}+\sum_{m, n=1}^{\infty} C_{2 m, 2 n}^{3} a_{2 m} a_{2 m} b_{2 n}\right] \\
& -\frac{\rho^{3}}{4}\left[D^{0}+\sum_{n=1}^{\infty} D_{2 n}^{1} b_{2 n}+\sum_{n=1}^{\infty} D_{2 n}^{1} a_{2 n} a_{2 n}+\sum_{m, n, p=1}^{\infty} D_{2 m, 2 n, 2 p}^{3} a_{2 m} a_{2 n} a_{2 p}+\sum_{m, n=1}^{\infty} D_{2 m, 2 n}^{4} a_{2 m} a_{2 m} b_{2 n}\right. \\
& \left.+\sum_{m, n, p, q=1}^{\infty} D_{2 m, 2 n, 2 p, 2 q}^{5} a_{2 m} a_{2 n} a_{2 p} b_{2 q}\right]
\end{aligned}
$$


and the equation of state is given by

$$
\begin{aligned}
\frac{P}{\rho k_{B} T}= & -\frac{\rho}{2}\left[B^{0}+\sum_{n=1}^{\infty} B_{2 n}^{1} b_{2 n}\right]-\frac{2 \rho^{2}}{3}\left[C^{0}+\sum_{n=1}^{\infty} C_{2 n}^{1} b_{2 n}+\sum_{m=1}^{\infty} C_{2 m}^{2} a_{2 m} a_{2 m}+\sum_{m, n=1}^{\infty} C_{2 m, 2 n}^{3} a_{2 m} a_{2 m} b_{2 n}\right] \\
& -\frac{3 \rho^{3}}{4}\left[D^{0}+\sum_{n=1}^{\infty} D_{2 n}^{1} b_{2 n}+\sum_{n=1}^{\infty} D_{2 n}^{1} a_{2 n} a_{2 n}+\sum_{m, n, p=1}^{\infty} D_{2 m, 2 n, 2 p}^{3} a_{2 m} a_{2 n} a_{2 p}+\sum_{m, n=1}^{\infty} D_{2 m, 2 n}^{4} a_{2 m} a_{2 m} b_{2 n}\right. \\
& \left.+\sum_{m, n, p, q=1}^{\infty} D_{2 m, 2 n, 2 p, 2 q}^{5} a_{2 m} a_{2 n} a_{2 p} b_{2 q}\right] .
\end{aligned}
$$

\section{APPENDIX B: GOVERNING EQUATIONS FOR MOLECULAR DYNAMICS SIMULATIONS}

Since the molecules are anisotropic, the center of mass position and orientation of each are given by

$$
\begin{gathered}
\vec{r}(t)=\vec{r}(0)+\vec{v}(0) t, \\
\vec{u}(t)=\cos [\theta(t)] \widehat{i}+\sin [\theta(t)] \widehat{j},
\end{gathered}
$$

where $\theta(t)=\theta(0)+\omega t$ (with $\dot{\theta} \omega)$, so that

$$
\vec{u}(t)=\vec{u}(0) \cos [\omega(0) t)]+\frac{\vec{\omega}(0)}{\omega(0)} \times \vec{u}(0) \sin [\omega(0) t]
$$

Here $\vec{r}$ and $\vec{v}$ are the position and velocity of the center of mass, respectively, $\vec{u}$ is a unit vector indicating the orientation of the anisotropic molecule, and $\vec{\omega}=|\vec{\omega}| \hat{k}$ is its angular velocity. The above two equations describe the time evolution of a molecule in its free-moving state. However, it is inevitable that a collision with another molecule will occur. We consider only two-body collisions, since it is rare for three bodies to collide simultaneously. When a collision occurs, it punctuates the free motion of the molecule and the time evolution as expressed by Eq. (B1) has to start again. Hence in Eq. (B1) the time 0 may be regarded as the instant of the last collision. In order to simulate the system's dynamics, it is important to (1) find the time duration to the next collision, and (2) find the linear and angular momenta change caused by the collision. For (2), one can appeal to the energy and momentum conservation laws, which states that for two colliding molecules $i$ and $j$,

$$
\begin{gathered}
m \vec{v}_{i}^{\prime}=m \vec{v}_{i}+P_{i j} \vec{n}, \quad m \vec{v}_{j}^{\prime}=m \vec{v}_{j}-P_{i j} \vec{n}, \\
I \vec{\omega}_{j}^{\prime}=I \vec{\omega}_{j}-\vec{r}_{j} \times P_{i j} \vec{n}, \quad I \vec{\omega}_{i}^{\prime}=I \vec{\omega}_{i}+\vec{r}_{i} \times P_{i j} \vec{n},
\end{gathered}
$$

where $I$ denotes the moment of inertia, and the momentum change $P_{i j}$ may be obtained by adding the following condition:

$$
\begin{aligned}
& \frac{1}{2} m\left(\vec{v}_{i}^{2}+\vec{v}_{j}^{2}\right)+\frac{1}{2} I\left(\vec{\omega}_{i}^{2}+\vec{\omega}_{j}^{2}\right) \\
& \quad=\frac{1}{2} m\left(\vec{v}_{i}^{\prime 2}+\vec{v}_{j}^{\prime 2}\right)+\frac{1}{2} I\left(\vec{\omega}_{i}^{\prime 2}+\vec{\omega}_{j}^{\prime 2}\right) .
\end{aligned}
$$

By substituting Eqs. (B4) and (B5) into Eq. (B6), we obtain

$$
P_{i j}=\frac{-\vec{n} \cdot\left(\vec{v}_{i}-\vec{v}_{j}+\vec{\omega}_{i} \times \vec{r}_{i}-\vec{\omega}_{j} \times \vec{r}_{j}\right)}{\frac{1}{m}+\frac{1}{2 I}\left[\left(\vec{r}_{i} \times \vec{n}\right)^{2}+\left(\vec{r}_{j} \times \vec{n}\right)^{2}\right]} .
$$

For (1), finding the time to the next collision $t$, we use the condition that

$$
\left|\vec{\rho}_{i}(t)-\vec{\rho}_{j}(t)\right|^{2}-1=0,
$$

and

$$
\vec{\rho}_{i}(t)=\vec{r}_{i}(t)+\vec{u}_{i}(t) \Delta r_{i}(t), \quad \vec{\rho}_{j}(t)=\vec{r}_{j}(t)+\vec{u}_{j}(t) \Delta r_{j}(t),
$$

where $t=0$ is defined to be the time of the previous step, just before the collision, the vectors $\vec{\rho}_{i, i}(t)$ denote the positions for the centers of the two colliding disks, and $\Delta r_{i, j}(t)$ are their respective distances from the centers of mass of the two molecules. To find the two relevant disks that will collide, one just has to move the time one step forward. If the two molecules overlap, then go back to the previous step, and the two overlapping disks are the relevant disks to be considered. (See Fig. 7.) From the previous equations, it follows that

$$
\begin{aligned}
\vec{\rho}_{i}(t)-\vec{\rho}_{j}(t)= & \vec{r}_{i}(t)+\vec{u}_{i}(t) \Delta r_{i}-\vec{r}_{j}(t)-\vec{u}_{j}(t) \Delta r_{j} \\
= & \left(\vec{r}_{i}-\vec{r}_{j}\right)+\left(\vec{v}_{i}-\vec{v}_{j}\right) t+\left[\vec{u}_{i} \Delta r_{i} \cos \left(\omega_{i} t\right)\right. \\
& \left.-\vec{u}_{j} \Delta r_{j} \cos \left(\omega_{j} t\right)\right] \\
& +\left[\frac{\vec{\omega}_{i}}{\omega_{i}} \times \vec{u}_{i} \Delta r_{i} \sin \left(\omega_{i} t\right)\right. \\
& \left.-\frac{\vec{\omega}_{j}}{\omega_{j}} \times \vec{u}_{j} \Delta r_{j} \sin \left(\omega_{j} t\right)\right] .
\end{aligned}
$$

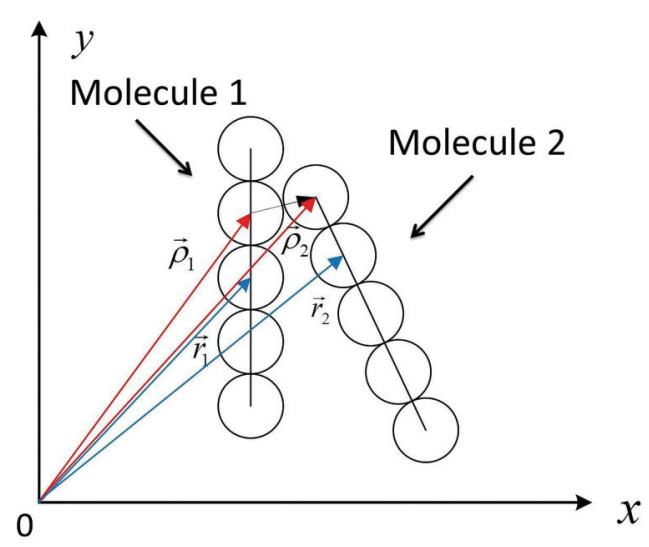

FIG. 7. (Color online) A diagram illustrating two colliding molecules with aspect ratio 5 . Here $\vec{r}$ denotes the center of mass, and $\vec{\rho}$ denotes the center of the disk that is in contact with the other molecule. The black arrow denotes the difference $\vec{\rho}_{2}(t)-\vec{\rho}_{1}(t)$, the magnitude of which is to be compared with the disk diameter. 
By regarding $\omega_{i, j} t \ll 1$, we expand $\cos \left(\omega_{i, j} t\right)$ and $\sin \left(\omega_{i, j} t\right)$ to sixth and fifth order in $\omega_{i, j} t$, respectively. Then Eq. (B8) becomes a sixth-order polynomial equation for $t$. We take the smallest solution to be the time to collision.
To start the simulation, we use random configurations for low densities, and use ordered "crystalline" configurations for high densities. About 1000 molecules were used in the simulations, with a periodic boundary condition.
[1] L. Onsager, Ann. N.Y. Acad. Sci. 51, 627 (1949).

[2] Introduction to Liquid Crystals, edited by E. B. Priestley, P. J. Wojtowicz, and P. Sheng (Plenum, New York, 1974).

[3] P. G. de Gennes, The Physics of Liquid Crystals (Oxford University Press, London, 1974).

[4] I. W. Steward, The Static and Dynamic Continuum Theory of Liquid Crystals (Taylor \& Francis, London, 2004).

[5] Y.-Z. Xie, The Physics of Liquid Crystals (Scientific Press, Beijing, 1988) (in Chinese).

[6] S. Chandrasekhar, Liquid Crystals (Cambridge University Press, Cambridge, UK, 1977).

[7] D. Frenkel, J. Chem. Phys. 91, 4912 (1987).

[8] P. Sheng, J. Chem. Phys. 59, 1942 (1973).

[9] G. R. Luckhurst, C. Zannoni, Nature 267, 412 (1977).

[10] W. Maier and A. Saupe, Z. Naturforsch. 15a, 287 (1960).

[11] A. D. Vries, Mol. Cryst. Liq. Cryst. 10, 31 (1970).

[12] D. Droulias, A. G. Vanakaras, and D. J. Photinos, Liq. Cryst. 37, 969 (2010).

[13] E. T. Samulski, Liq. Cryst. 37, 669 (2010).

[14] O. Francescangeli, V. Stanic, S. I. Torgova, A. Strigazzi, N. Scaramuzza, C. Ferrero, I. P. Dolbnya, T. M. Weiss, R. Berardi, L. Muccioli, S. Orlandi, and C. Zannoni, Adv. Funct. Mater. 19, 2592 (2009).

[15] L. Joshi, S.-W. Kang, D. M. Agra-Kooijman, and S. Kumar, Phys. Rev. E 80, 041703 (2009).
[16] H.-S. Park, S.-W. Kang, L. Tortora, Y. Nastishin, D. Finotello, S. Kumar, and O. D. Lavrentovich, J. Phys. Chem. B 112, 16307 (2008).

[17] P. Sheng and P. J. Wojtowicz, Phys. Rev. A 14, 1883 (1976).

[18] R. Tao, P. Sheng, and Z. F. Lin, Phys. Rev. Lett. 70, 1271 (1993).

[19] K. Huang, Statistical Mechanics (Wiley, New York, 1967), p. 297.

[20] V. V. Ginzburg, M. A. Glaser, and N. A. Clark, Liq. Cryst. 21, 265 (1996).

[21] J. M. Kosterlitz and D. Thouless, J. Phys. C 6, 1181 (1973).

[22] M. Allen and D. Tildesley, Computer Simulation of Liquids (Clarendon, New York, 1987).

[23] D. C. Rapaport, The Art of Molecular Dynamics Simulation (Cambridge University Press, Cambridge, UK, 2004).

[24] D. W. Rebertus and K. M. Sando, J. Chem. Phys. 67, 2585 (1977).

[25] M. P. Allen, D. Frenkel, and J. Talbot, Comput. Phys. Rep. 9 301 (1989).

[26] A. Donev, S. Torquato, and F. H. Stillinger, J. Comput. Phys. 202, 737 (2005).

[27] B. I. Halperin and D. R. Nelson, Phys. Rev. Lett. 41, 121 (1978).

[28] E. P. Bernard and W. Krauth, Phys. Rev. Lett. 107, 155704 (2011).

[29] Y. Peng, Z. Wang, A. M. Alsayed, A. G. Yodh, and Y. Han, Phys. Rev. Lett. 104, 205703 (2010). 